\title{
Leishmaniose mit kutaner und viszeraler Beteiligung bei einem 13 Monate alten Jungen
}

\author{
Eva Landmann¹, C. Bogdan², N. Donhauser ${ }^{2}$, A. Artlich¹, B. Staude³, L. Gortner ${ }^{1}$ \\ ${ }^{1}$ Zentrum für Kinderheilkunde, Abt. Allgemeine Pädiatrie und Neonatologie, Justus-Liebig-Universität, Gießen \\ 2 Institut für Klinische Mikrobiologie, Immunologie und Hygiene der Universität Erlangen-Nürnberg \\ ${ }^{3}$ Praxis für Kinderheilkunde, Gießener Straße 31, 35457 Lollar
}

\section{Zusammenfassung}

Leishmaniosen sind durch Protozoen der Gattung Leishmania hervorgerufene Erkrankungen, die die inneren Organe oder die Haut und/ oder die Schleimhäute betreffen. Während die benigne kutane Form selbstlimitierend ist, verläuft die viszerale Form (Kala-Azar) unbehandelt in $80 \%$ der Fälle tödlich. Bei dem Symptomenkomplex Fieber, Hepatosplenomegalie und Panzytopenie sollte bei spezifischer Auslandsanamnese eine Kala-Azar ausgeschlossen werden. Wir berichten über einen 13 Monate alten Jungen mit kombinierter Hautund viszeraler Manifestation.

\section{Leishmaniasis with cutaneous and visceral involvement in a 13-month-old boy}

Leishmaniasis is an anthropozoonosis caused by infection with leishmania parasites with either cutaneous, mucosal or visceral (kalaazar) involvement. While the benign cutaneous form is self-limited death occurs in approximately $80 \%$ of children with kala-azar when untreated. The diagnosis of kala-azar should not be missed in children presenting with fever, hepatosplenomegaly and pancytopenia especially with a history of sand fly bites. We report the case of a 13-month-old boy with both cutaneous and visceral involvement.

\section{Fallbericht}

Anamnese: Vier Monate vor stationärer Aufnahme traten rötliche Papeln am Kopf und Hals des Kindes auf. Es war zunehmend müde und fieberte seit einem Monat intermittierend bis $40,5^{\circ} \mathrm{C}$. Bei Anämie und Hepatosplenomegalie erfolgte die Einweisung unter der Verdachtsdiagnose Kala-Azar. Sechs Wochen vor Beginn der Symptomatik sei das Kind auf Malta von Sandmücken gestochen worden.

Untersuchungsbefund: Blasser $1 \frac{1}{12}$ Jahre alter Junge, reduzierter Allgemein- und guter Ernährungszustand, Körpertemperatur $38,8^{\circ} \mathrm{C}$, an Kopf und Hals zehn rötliche Papeln (Durchmesser bis $0,5 \mathrm{~cm})$, z.T. zentral ulzerierend oder mit gelblichen Krusten belegt (Abb.1), Milz $5 \mathrm{~cm}$, Leber $4 \mathrm{~cm}$ unter dem Rippenbogen palpabel.

Blutbild und pathologische Laborbefunde: Leukozyten 5,2 G/l (Segmentkernige 0,52 G/I, Stabkernige 1,61 G/I, Lymphozyten 2,34 G/l,

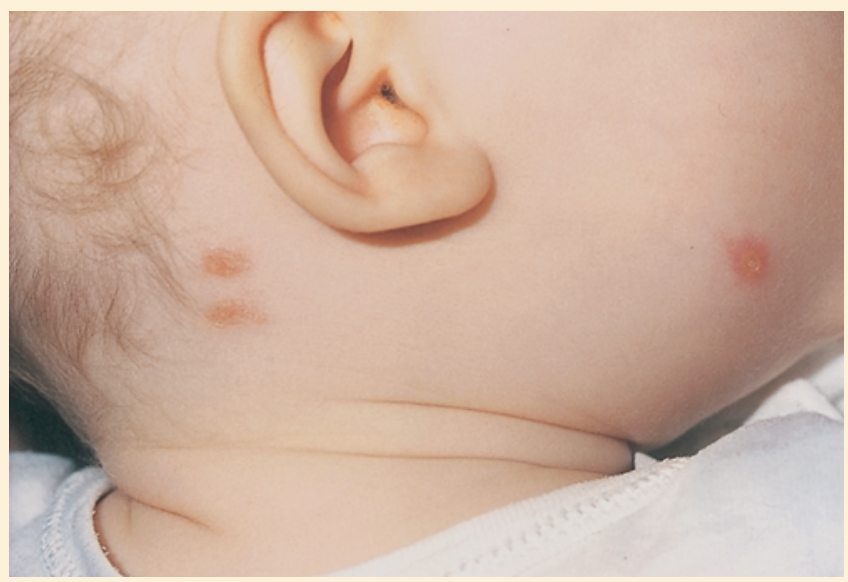

Abb.1 Hautbefund vor Behandlung. An exponierten Hautstellen finden sich papulöse Effloreszenzen, die z.T. ulzerieren und mit gelblichen Krusten belegt sind.
Monozyten 0,52 G/I, Eosinophile 0,05 G/I), Erythrozyten 3,6 T/I, Hämoglobin $75 \mathrm{~g} / \mathrm{l}$, Hämatokrit 24\%, MCV $67 \mathrm{fl}, \mathrm{MCH} 21,1 \mathrm{pg}$, Thrombozyten $213 \mathrm{G} / \mathrm{l}$, Retikulozyten 19\%, GOT $31 \mathrm{U} / \mathrm{l}$, Cholinesterase 2179 U/I, LDH 1356 U/l, IgG 18,5 g/l, CRP 29,2 mg/l.

Spezifische Diagnostik: Leishmanien-Antikörper (IFT) im Serum positiv, Nachweis von Leishmania Amastigoten (Abb. 2) und Leishmania-DNA (PCR) im Knochenmark, im Hautbiopsat Nachweis von Leishmania Amastigoten, Leishmania-DNA (PCR) und Anzucht von Leishmania Promastigoten.

\section{Diagnose: Leishmaniose mit kutaner und viszeraler Beteiligung}

Therapie und Verlauf: Wir behandelten mit liposomalem Amphotericin B $(3 \mathrm{mg} / \mathrm{kg} \mathrm{KG} /$ die, 10 Tage intravenös). Unter Therapie kam es rasch zur Entfieberung und die Blutbildveränderungen, Transaminasenerhöhung und Hepatosplenomegalie waren rückläufig. Vier Tage nach Therapieende hatte sich das Blutbild

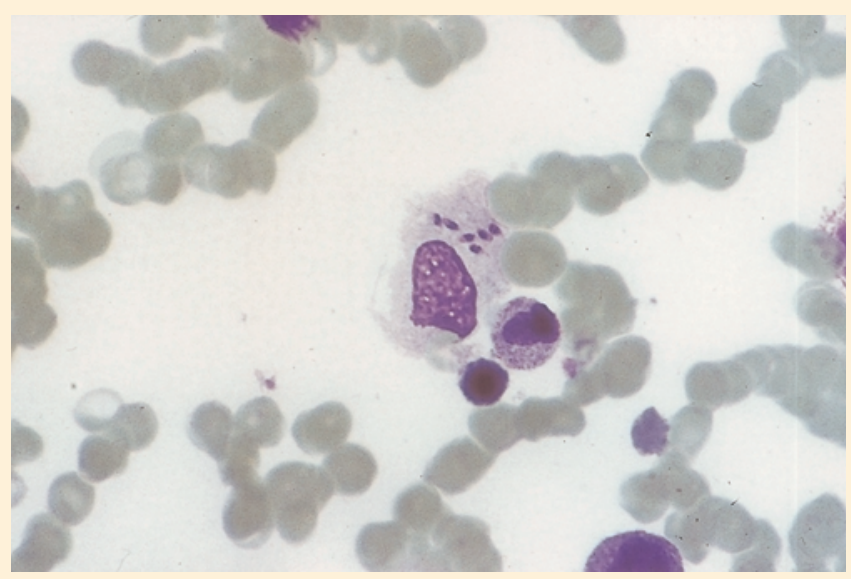

Abb. 2 Knochenmark: Mikroskopischer Nachweis von LeishmaniaAmastigoten (Giemsafärbung). 
normalisiert. Zwei Monate nach Therapieende bestanden noch in der Größe deutlich rückläufige jetzt makulöse Effloreszenzen.

\section{Kommentar}

Ätiologie, Epidemiologie und Pathogenese: Leishmaniosen sind durch verschiedene Spezies der Gattung Leishmania verursachte Krankheitsbilder, die im Mittelmeerraum und in weiten Gebieten der tropischen und subtropischen Zone verbreitet sind. Man unterscheidet Verläufe mit Befall der viszeralen Organe (KalaAzar), kutane (Orientbeule, diffuse kutane Leishmaniose) und mukokutane Formen. Der Krankheitsverlauf hängt von der Parasitenspezies und der Immunitätslage des Wirts ab [1,2]. In Deutschland erkranken jährlich etwa 200 Patienten an einer Leishmaniose, davon mindestens zehn an einer Kala-Azar. Betroffen sind v.a. Kinder bis zum Schulalter $[3,4]$. Es handelt sich bei uns meist um aus dem Mittelmeerraum importierte Erkrankungen. Dort stellen v. a. Hunde das Reservoir dar; als Vektor dienen Sandmücken. Der Mensch wird akzidentell infiziert.

Bei der viszeralen Leishmaniose werden die Leishmanien nach Inokulation in die Haut durch Makrophagen und dendritische Zellen phagozytiert und im Organismus verbreitet. Abhängig von der zellulären Immunitätslage [2] bleibt die Infektion asymptomatisch oder es treten unspezifische Krankheitszeichen auf, die ohne Therapie ausheilen. Bei rund einem Viertel der Infizierten entwickelt sich das Vollbild der Kala-Azar.

Bei der kutanen Leishmaniose akkumulieren an der Infektionsstelle Lymphozyten um die erregerhaltigen Makrophagen, was zur Entstehung von gemischtzelligen Granulomen und somit vermutlich zur lokalen Begrenzung der Infektion führt.

Symptome: Nach einer Inkubationszeit von Wochen bis Monaten entwickeln sich bei der viszeralen Form die in Tabelle 1 aufgeführten Symptome. Bei der benignen kutanen Leishmaniose treten typischerweise an unbedeckten Hautpartien juckende Papeln auf, die im Verlauf ulzerieren und nach Monaten narbig abheilen.

Tab. 1 Klinische und laborchemische Befunde bei viszeraler Leishmaniose

Befunde bei Verdacht auf viszerale Leishmaniose

Klinik:

- Fieber ohne Peridiodizität

- Hepatosplenomegalie (oft ausgeprägt)

- Müdigkeit

- Generalisierte Lymphadenopathie (fakultativ)

- Spätstadium: starker Gewichtsverlust, generalisierte Ödeme, Hämorrhagien und schwere Infektionen

Labor:

- Anämie, Leukozytopenie mit Neutropenie und Eosinopenie, evtl. Thrombozytopenie

- BSG-Beschleunigung

- lgG-Erhöhung

- Transaminasenerhöhung (meist leicht)

Die bei unserem Patienten bestehende kutane und viszerale Leishmaniose ist nach unserem Kenntnisstand bei einem immunkompetenten Patienten bisher noch nicht beschrieben.

Diagnostik: Die Untersuchungen (s. Tabelle 2) sollten in der spezifischen Diagnostik erfahrenen Untersuchern vorbehalten sein.
Tab. 2 Diagnostik bei Verdacht auf Leishmaniose

Diagnostik bei Verdacht auf kutane Leishmaniose

Hautbiopsat: Nachweis von Leishmanien (Immunhistologie, HE-Färbung), und/oder von Leishmanien-DNA (PCR), Anzucht von Leishmanien

Serum: Nachweis von Anti-Leishmanien-Antikörpern mittels IFT oder Western-Blot (bei Einzelläsion meist negativ)

\section{Diagnostik bei Verdacht auf viszerale Leishmaniose}

Knochenmarkspunktat: Goldstandard ist der Erregernachweis im Knochenmark (Immunhistologie, Giemsa-Färbung, Kultur und/oder PCR)

Serum: Nachweis von Anti-Leishmanien-Antikörpern (IFT)

\section{Differentialdiagnose: Siehe hierzu Tab. 3.}

Therapie: Neben der bislang etablierten Therapie mit pentavalenten Antimonpräparaten wird aufgrund besserer Therapieerfolge, einer kürzeren Therapiedauer und geringerer Nebenwirkungen zunehmend liposomales Amphotericin B eingesetzt. In einer Dosierung von $3 \mathrm{mg} / \mathrm{kgKG} / \mathrm{d}$ über 10 Tage wurden bisher keine Therapieversager beschrieben [5].

Tab. 3 Differentialdiagnose der viszeralen und kutanen Leishmaniose

\section{Differentialdiagnose der Leishmaniose}

Kutane Form: Superinfizierte Verletzungen oder Mückenstiche, Neoplasien der Haut, Infektionen mit atypischen Mykobakterien, Hauttuberkulose, Lepra und Syphilis.

Viszerale Form: Leukämie, Lymphome, EBV-Infektion, Malaria, Tuberkulose, Sarkoidose, Schistosomiasis, Brucellose und Typhus.

\section{Literatur}

${ }^{1}$ Bogdan G, Gessner A, Röllinghoff M. Cytokines in Leishmaniasis: a complex network of stimulatory and inhibitory interactions. Immunobiol 1993; 189: 356-396

${ }^{2}$ Bogdan C, Röllinghoff $M$. The immune response to leishmania: mechanisms of parasite control and evasion. Int J Parasitol 1998; 28: $121-134$

${ }^{3}$ Epidemiologisches Bulletin des Robert-Koch-Instituts. Ausgabe Nr. 25, 23. Juni 2000, Seite 201

${ }^{4}$ Deutsche Gesellschaft für Pädiatrische Infektiologie e.V. (DGPI), Arbeitsgruppe „Parasitologie“. Leishmaniosen. Klin Pädiatr 1998; 210: $428-429$

${ }^{5}$ Davidson RN, di Martino L, Gradoni L, Giacchino R, Gaeta GB, Pempinello R, Scotti S, Cascio A, Castagnola E, Maisto A, Gramiccia M, Caprio D, Wilkinson RJ, Bryceson AD. Short-course treatment of visceral leishmaniasis with liposomal amphotericin B (AmBisome). Clin Infect Dis 1996; 22: $938-943$

\section{Dr. med. Eva Landmann}

Zentrum für Kinderheilkunde Justus-Liebig-Universität

Feulgenstraße 12

35392 Gießen

E-mail: Eva.Landmann@paediat.med.uni-giessen.de 